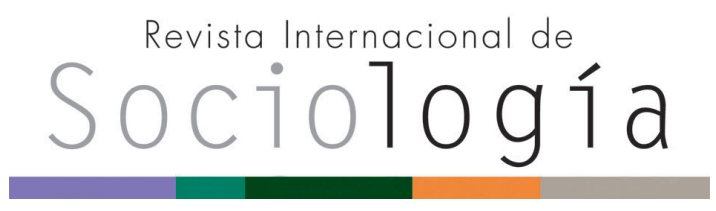

Revista Internacional de Sociología RIS

vol. 78 (1), e146, enero-marzo, 2020, ISSN-L:0034-9712 https://doi.org/10.3989/ris.2020.78.1.18.064

\section{SUSTENTACIÓN PRINCIPAL DEL HOGAR EN EL GRUPO DE EDAD DE 25 A 34 AÑOS EN ESPAÑA (2006, 2011 Y 2015)}

\author{
MARTA DONAT LÓPEZ \\ Universidad de Granada \\ martalou89@correo.ugr.es \\ ORCID iD: https://orcid.org/0000-0002-6323-2779
}

\author{
Ma DoLORES MARTíN-LAGos LÓPEZ \\ Universidad de Granada \\ Imlagos@ugr.es \\ ORCID iD: https://orcid.org/0000-0003-3540-9079
}

\author{
ELISABETH MOLES LÓPEZ \\ Universidad de Granada \\ elisabethmoles@ugr.es \\ ORCID iD: https://orcid.org/0000-0001-6586-2654
}

Cómo citar este artículo / Citation: Donat López, M., M. D. Martín-Lagos López y E. Moles López. 2020. "Sustentación principal del hogar en el grupo de edad de 25 a 34 años en España (2006, 2011 y 2015)". Revista Internacional de Sociología 78(1): e146. https://doi. org/10.3989/ris.2020.78.1.18.064

\section{RESUMEN}

Se propone conocer la influencia de ciertas variables socioeconómicas sobre la emancipación de los jóvenes dependiendo de si éste es sustentador principal de su hogar, o bien si es hijo o hija del mismo. Con ello se realiza una aproximación a la emancipación de los jóvenes. Tomando como referencia tres fechas concretas (2006, 2011 y 2015) se persigue constatar en qué medida afecta la recesión económica. Se utilizan análisis descriptivos y regresiones logísticas binarias usando la Encuesta de Presupuestos Familiares. Existen algunas diferencias dependiendo del año, así como también ciertas tendencias generales. A mayor nivel de estudios, es más probable que un joven viva con su sustentador principal, mientras que ser varón, tener entre 30 y 34 años o percibir un salario mayor de $1000 €$ se aproxima más al perfil de "cabeza de familia". En cuanto a los sustentadores principales, un varón de 30 a 34 años con un nivel de ingresos mayor de $1000 €$ es el perfil más próximo al contrato indefinido.
\end{abstract}

\section{Palabras Clave}

Cabeza de familia; Crisis económica; Emancipación; España; Juventud.

\section{MAIN HOUSEHOLD BREADWINNERS AGED BETWEEN 25 AND 34 YEARS OLD IN SPAIN (2006, 2011 AND 2015)}

Copyright: (C) 2020 CSIC. Este es un artículo de acceso abierto distribuido bajo los términos de la licencia de uso y distribución Creative Commons Reconocimiento 4.0 Internacional (CC BY 4.0).

Recibido: 26/04/2018. Aceptado: 11/02/2019 Publicado online: 16/03/2020

\begin{abstract}
It is proposed to know the influence of certain socioeconomic variables depending on whether a young person is the main breadwinner of his or her household, or the child of the main breadwinner. In this way, an approximation is made to the independence of youth. Taking three specific dates as a reference -2006, 2011 and 2015-, the aim is to ascertain the extent to which the economic recession is affecting them. Descriptive analyses and binary logistic regressions have been done, using the Household Budget Survey. There are some differences depending on the year, as well as some general trends. A higher level of education increases the probability that a youth is the child of the main breadwinner of the household, while being male, between 30 and 34 years old or receiving a salary greater than $€ 1,000$ is closer to the profile of breadwinner. As for the main breadwinners, a male aged 30 to 34 with an income level greater than $€ 1,000$ is the profile closest to a permanent contract.
\end{abstract}

\section{KEYWORDS}

Breadwinner; Economic crisis; Independence; Spain; Youth. 


\section{INTRODUCCIÓN}

En la época de prosperidad económica en España, la emancipación fue un fenómeno social creciente, tanto por el número de jóvenes que llevaban este proceso a cabo como por la edad, cada vez más temprana, en la que se producía. Sin embargo, desde que comenzó a manifestarse la crisis económica en 2008, esta tendencia se ha ralentizado. En el cuarto trimestre de 2004 el número de jóvenes que abandonaron el hogar de sus progenitores se incrementó en un 5,6 \% respecto al año anterior; a finales de 2008, este aumento fue tan solo del 1,5 \% (Observatorio de Emancipación 2009). Esta tendencia, en línea con las investigaciones de Gentile (2010; 2015; 2016) y Ahn y Sánchez-Marcos (2017), permite afirmar que la emancipación es un fenómeno dependiente de los ciclos económicos.

Por otra parte, a los ciclos económicos hay que sumarles la privatización de las empresas públicas y la flexibilización del empleo, consecuencias de la globalización de las economías occidentales (Jurado 2007). Esto ha dado lugar a una precarización de las condiciones laborales y a un aumento de la incertidumbre, lo que afecta en mayor medida a los jóvenes. Estas dificultades en el empleo obstaculizan la emancipación, ya que esta necesita de cierta solidez económica. Por ello, la estabilidad económica y laboral están relacionadas con el logro de una autosuficiencia económica y de la formación de una nueva unidad familiar o un nuevo hogar (Blossfeld et al. 2006).

Basándose en la importancia de investigaciones anteriores (Blossfeld et al., 2006; Gentile, 2015; 2016; Ahn y Sánchez-Marcos, 2017), este artículo se centra en analizar la precariedad laboral y la autosuficiencia de los jóvenes mediante variables como el tipo de contrato y el nivel de ingresos, entre otras.

El acercamiento a estos resultados resulta crucial para el desarrollo de un diagnóstico aproximado sobre la situación de los jóvenes, lo que puede contribuir a la elaboración de políticas en materia de juventud. Como objetivos, se pretende conocer la influencia de ciertas variables socioeconómicas, dependiendo de si un joven es sustentador principal de su hogar, o bien si es hijo o hija del mismo y, de esta forma, llevar a cabo una aproximación a la emancipación de los jóvenes, dibujando una serie de perfiles. Además, tomando como referencia tres fechas concretas (2006, 2011 y 2015), se persigue constatar si las líneas generales del perfil de estos jóvenes cambian o divergen por fecha, viendo así en qué medida afecta la recesión económica a todo ello, observando tres momentos: "antes, durante y después" de dicha crisis. No se trata de una evolución temporal de los jóvenes de la muestra, sino más bien se podría definir como un análisis de tres fotografías independientes, separadas en el tiempo; se está tratando una encuesta transversal.

En definitiva, tanto la situación laboral como las condiciones socioeconómicas de los jóvenes, lejos de proporcionar un equilibrio laboral y económico, no siempre permiten que estos tengan una estabilidad económica para tomar la iniciativa de formar un hogar propio. Este contexto de incertidumbre aportado por la literatura requiere de una concreción en términos cuantitativos, objeto del presente artículo.

Para llevar a cabo el análisis se emplea la Encuesta de Presupuestos Familiares. Esta base de datos se suele utilizar para llevar a cabo estudios relativos a cuestiones de consumo, como son los gastos desagregados en función del bien adquirido por hogar. No obstante, y como novedad en el ámbito de la investigación, en este artículo se considera el aprovechamiento del resto de información de la misma para, de esta forma, efectuar una aproximación a la emancipación de los jóvenes.

Otra cuestión esencial que aporta este estudio radica en el hecho de que se analiza la emancipación a partir de la autosuficiencia económica de los jóvenes. Así, se utiliza el término emancipación no solo para hacer referencia al abandono, por parte de los jóvenes, del hogar de los progenitores para formar un hogar propio. La emancipación domiciliar o residencial se concibe como "el paso de la dependencia de vivir en casa de los padres a la autonomía de vivir separado de estos en una casa propia" (Miret 2007: 302). Por tanto, este estudio no tiene en cuenta la emancipación únicamente como el hecho del abandono del hogar de los progenitores y la creación de un hogar propio, sino que también se incorpora la autosuficiencia económica a la hora de hablar de la misma.

Como modo de aproximación a la emancipación desde la autosuficiencia económica, se compara la situación en el hogar como sustentador principal o como hijo o hija del sustentador principal, entendiendo el hecho de ser el sustentador principal del hogar como equiparable al de ser económicamente autosuficiente y, por ende, al de estar emancipado; así como el hecho de ser hijo o hija del sustentador principal del hogar se considera equiparable al de no estar emancipado. La relación de estos respecto al sustentador principal del hogar en cuestión parece ser un buen predictor para ello, puesto que se construye a partir de la variable "ingresos". Por otro lado, esta encuesta brinda la posibilidad de comparar tres fechas relacionadas con la recesión económica en España, lo que permite indagar en algunos cambios que afectan a esa autosuficiencia económica de los jóvenes.

A continuación, se desarrolla un marco analítico que contextualiza la temática de la investigación en España, así como también los resultados de la literatura sobre la emancipación en este país. Posteriormente, se enuncian los objetivos e hipótesis, así como también la metodología utilizada. Posteriormente, se exponen los resultados más significativos en función de los objetivos e hipótesis de la investigación para, finalmente, extraer conclusiones y recomendaciones para futuras líneas de investigación. 


\section{La situación laboral de los jóvenes en España y su relación con la autosuficiencia económica}

Para llevar a cabo un análisis de la autosuficiencia económica de los jóvenes, inicialmente se define lo que se entiende por precariedad laboral. En este estudio se atiende a la definición dada por Vosko (2010), que acuña este término para todo aquel empleo caracterizado por un alto nivel de incertidumbre derivada de unos salarios bajos y unos beneficios sociales y derechos legales limitados.

A la hora de analizar dicha precariedad en España, es necesario contextualizar las múltiples transformaciones que se han producido en las últimas décadas. Numerosos cambios educativos, laborales y políticos han afectado a la situación de los jóvenes.

En el ámbito educativo, ha tenido lugar una ampliación de la etapa formativa. Esta prolongación del periodo de implicación de los jóvenes en el sistema educativo, caracterizado por el hecho de que en algunas ocasiones se compagina formación y empleo, tiene como consecuencia el retraso en la incorporación al mercado laboral, cuyo fin es una mejor preparación para esta nueva etapa (Jones 1995; Garrido, Requena y Rivière 1995; Fernández, 1997; García y Merino 2006; Miret 2006; García-Montalvo 2009). Respecto al nivel de estudios como predictor de la autosuficiencia de los jóvenes, Miret (2006) señala que una prolongación de la etapa formativa no implica una variación en el calendario emancipatorio.
Por otra parte, una serie de reformas laborales, cuyo propósito era combatir el desempleo y lograr un alto nivel de competitividad de las empresas, han supuesto la merma de algunos derechos y de la estabilidad de los trabajadores. En la tabla 1 se pueden apreciar los cambios en el ámbito laboral que han supuesto las principales reformas llevadas a cabo en España desde 1984 a 2012, periodo que abarca la época anterior, el inicio y el desarrollo de la crisis económica (Hernández 2012a). A lo largo de estas fechas se observa que se han producido variaciones significativas que afectan de un modo $u$ otro a los jóvenes, como puede ser la limitación del contrato temporal (2010), el abaratamiento del despido y la ampliación en la edad del uso del contrato de formación (2012), entre otras.

A todo lo anteriormente expuesto hay que sumar que la precariedad en el empleo da lugar a que la transición a la vida adulta se encuentre sometida a diferentes periodos, en los que los jóvenes circulan por el mercado de trabajo en busca de esa estabilidad (Requena 2002; Snyder, 2014). Según datos del INE, en el primer trimestre del año 2017 el 22,9 \% de los jóvenes españoles de 25 a 34 años se encontraba en paro, doblando la media europea, la cual se sitúa en $11,2 \%$ (Eurostat, 2016). Además, como se puede observar en el gráfico 1, a pesar de la evolución positiva del Salario Mínimo Interprofesional (SMI) en España, la media del salario mensual de los jóvenes de entre 24 a 35 años ha disminuido en los últimos años, por lo que dicho colectivo resulta muy afectado por la citada precariedad.

Tabla 1.

Reformas laborales en España (1984-2012)

\begin{tabular}{|c|c|c|c|c|c|}
\hline $\begin{array}{l}\text { Ámbitos/ } \\
\text { Año reforma }\end{array}$ & 1984 & 1994 & 1997 & 2010 & 2012 \\
\hline Flexibilización & $\begin{array}{l}\text { Intensificación de la } \\
\text { flexibilidad laboral. }\end{array}$ & $\begin{array}{l}\text { Avance flexibilizador } \\
\text { en: tiempo de trabajo, } \\
\text { movilidad geográfica, } \\
\text { condiciones laborales } \\
\text { y estructura salarial. }\end{array}$ & & $\begin{array}{l}\text { Aumento de la } \\
\text { flexibilidad interna. }\end{array}$ & $\begin{array}{l}\text { Intensificación de } \\
\text { la flexibilidad, supo- } \\
\text { niendo un avance en } \\
\text { el proceso de desre- } \\
\text { gularización legal. } \\
\end{array}$ \\
\hline $\begin{array}{l}\text { Contratación } \\
\text { temporal o de } \\
\text { formación }\end{array}$ & $\begin{array}{l}\text { Eliminación de las } \\
\text { restricciones a la } \\
\text { contratación tempo- } \\
\text { ral de tipo no casual. }\end{array}$ & $\begin{array}{l}\text { Promoción de los } \\
\text { contratos formativos } \\
\text { o a tiempo parcial. }\end{array}$ & & $\begin{array}{l}\text { Limitación de la con- } \\
\text { tratación temporal. }\end{array}$ & $\begin{array}{l}\text { Ampliación de la } \\
\text { edad del uso del con- } \\
\text { trato de formación. }\end{array}$ \\
\hline $\begin{array}{l}\text { Contratación } \\
\text { indefinida }\end{array}$ & & & $\begin{array}{l}\text { Fomento contrata- } \\
\text { ción indefinida. }\end{array}$ & $\begin{array}{l}\text { Impulso de la contra- } \\
\text { tación indefinida. }\end{array}$ & \\
\hline Despido & & & $\begin{array}{l}\text { Abaratamiento del } \\
\text { despido para el } \\
\text { fomento de la con- } \\
\text { tratación indefinida. }\end{array}$ & $\begin{array}{l}\text { Favorecimiento del } \\
\text { despido colectivo } \\
\text { por crisis. }\end{array}$ & $\begin{array}{l}\text { Abaratamiento del } \\
\text { despido. }\end{array}$ \\
\hline Poder empresarial & & & & $\begin{array}{l}\text { Fomento y mejora } \\
\text { de la intermediación } \\
\text { laboral. }\end{array}$ & $\begin{array}{l}\text { Aumento del poder } \\
\text { empresarial. }\end{array}$ \\
\hline Social & & & & $\begin{array}{l}\text { Impulso del empleo } \\
\text { de los colectivos } \\
\text { vulnerables al des- } \\
\text { empleo. }\end{array}$ & \\
\hline
\end{tabular}

Fuente: Elaboración propia a partir de Hernández (2012a). 
Gráfico 1.

Evolución Salario Mínimo Interprofesional (SMI) y salario medio en España (2006-2017)

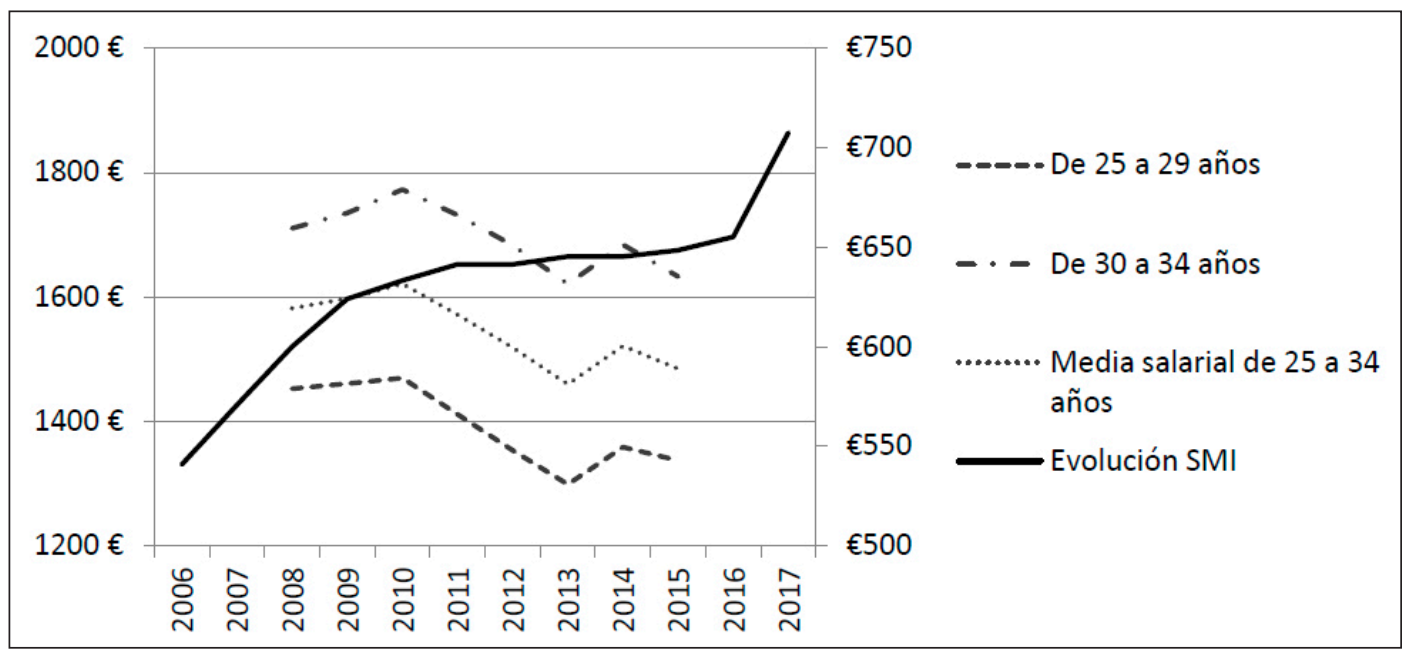

Fuente: Elaboración propia a partir de los datos del Ministerio de Empleo de España y del Instituto Nacional de Estadística. Eje de la derecha corresponde a la media de los salarios y eje de la izquierda corresponde a la evolución del SMI

En el año 2006, el salario medio de los jóvenes, según la Encuesta de Presupuestos Familiares, se situaba en 1008,98 euros mensuales. Lejos de lo que cabría esperar, teniendo en cuenta la evolución del SMI del total de la población, en una década el salario medio de los jóvenes ha descendido en 63,3 euros, situándose en 2015 en 949,68. Los jóvenes sustentadores principales percibían en 2006 un salario medio mensual de 1245,61 euros, el cual en 2015 ha pasado a ser de 1179,15 euros (INE 2006; 2015). Así, se puede observar la gran diferencia salarial que existe entre el grupo de los jóvenes y el conjunto de la población.

Esta situación de flexibilidad y precariedad laboral ha dado lugar a lo que Bourdieu denomina flexplotación: "un estado generalizado y permanente de inseguridad que tiende a obligar a los trabajadores a la sumisión, a la aceptación de la explotación" (Bourdieu 2000: 125-126), siendo los jóvenes uno de los grupos más vulnerables a esta situación (López 2005).

En definitiva, todas las cuestiones descritas, que implican un aumento en la precariedad laboral juvenil en España, denotan más dificultades a la hora de que un joven sea económicamente autosuficiente $y$, en consecuencia, de que se emancipe. Una vez expuestos los datos que revelan la situación de los jóvenes españoles en el periodo analizado, a continuación, se trata la repercusión de estos fenómenos en el retraso de la emancipación de los mismos, a la luz de las investigaciones existentes.

\section{Consecuencias de la precariedad laboral juvenil en la emancipación}

En los últimos años, diversos estudios han centrado su atención en los efectos que la precariedad o la in- certidumbre laboral tienen sobre los jóvenes, afectando a la emancipación y, en consecuencia, promoviendo su retraso (Garrido, Requena y Rivière 1995; Mitchell 2004; Moreno 2012a; South y Lei 2015; Burn y Szoeke 2016). La emancipación, vista como un fenómeno esencial en la transición que supone el paso de la juventud a la edad adulta, es una de las etapas más significativas en el ciclo vital de una persona, lo que suscita el interés de la investigación social (White 1994; Gee, Mitchell y Wister 1995; Berngruber 2015; Lary 2015). En los últimos años, esta fase de transición de una etapa a otra se caracteriza por unas trayectorias vitales cada vez más diversas e indefinidas. Estas transformaciones han generado un replanteamiento de la transición hacia los roles adultos, caracterizados por el cambio y el sondeo de posibles direcciones de la vida (Arnett, 2014).

En este sentido, Ahn y Sanchéz-Marcos (2017) destacan diversos factores que influyen en la emancipación de los jóvenes, entre los que se encuentran, por un lado, los de tipo económico. Los costes que la emancipación conlleva, como es la pérdida de poder adquisitivo frente al consumo, así como también los problemas de acceso a la vivienda, son buena muestra de ello (Ermisch y Di Salvo 1997; Ermisch 1999; Giannelli y Monfardini 2003; Lee y Painter 2013). Elementos como el desempleo (Martínez-Granado y Ruiz-Castillo 2002), la estabilidad laboral (Becker et al. 2010), el nivel de estudios (Melo y Miret 2010), el mayor acceso a préstamos económicos con el fin de paliar los gastos de la vivienda (Martins y Villanueva 2009) y la existencia de políticas sociales encaminadas al fomento de la emancipación (Aparicio-Fenoll y Oppedisano 2012) se señalan como condiciones económicas individuales (Aparicio-Fenoll y Oppedsano 2012; Lee y Painter 2013). Por otro lado, se señalan factores no económicos, como la protección parental, el mayor soporte económico (Manacorda y Moretti 2006), el sexo (Miret 2006; Moreno 
2012b; Pérez-Baleón 2012; Hernández y López 2015), el nivel de estudios (Shanahan 2000; Mykyta 2012) o la formación de pareja (Shanahan 2000; Mykyta 2012).

En España, la estabilidad económica y laboral, la unión afectiva con una pareja, el vínculo del matrimonio y la seguridad de una vivienda, preferiblemente en régimen de propiedad, determinan en gran medida la emancipación del joven (Shanahan 2000; lacovou 2010; Ayuso 2010; Mykyta 2012). De una parte, seguir en el hogar de los padres, facilita a los jóvenes, por un lado, lidiar con las dificultades actuales del mercado de trabajo y, por otro, aprovechar ese periodo para seguir formándose o buscar un trabajo que esté más acorde con sus capacidades y con su formación (López 2006; Vieira y Gamundi 2010).

Respecto a la estabilidad laboral y económica de los jóvenes, existen estudios que analizan la relación entre el tipo de empleo (temporal o indefinido) y la emancipación. Estos estudios, y como se observó en la investigación de Donat, Martín-Lagos y Moles que precede a este artículo, muestran que el nivel de ingresos y el tipo de contrato, en este caso indefinido, influyen de forma positiva en la probabilidad de emancipación de los jóvenes (Hidalgo y Pérez 2004). Por otro lado, encontrarse en situación de desempleo o tener un contrato temporal tendría una influencia negativa en la autosuficiencia de los jóvenes (Jurado 2006).

\section{Metodología}

\section{Información sobre la muestra y método de análisis}

Para conseguir los objetivos propuestos se ha utilizado una metodología cuantitativa. Se ha realizado una explotación de datos procedentes de la Encuesta de Presupuestos Familiares del INE, para los años 2006, 2011 y 2015.

En la selección de fechas a analizar se ha tenido en cuenta el año en el que se modificó la Encuesta de Presupuestos Familiares (2006), puesto que unas fechas anteriores no hubieran podido ser comparadas por cuestiones metodológicas. La finalidad de trabajar con datos de este periodo radica en que la crisis económica aún no se había manifestado y, por tanto, no constaban las últimas reformas laborales en España. Por otra parte, se ha seleccionado el año 2015 , fecha en la que ya aparecen síntomas claros de recuperación económica. Finalmente, se ha escogido el año 2011 como fecha intermedia, teniendo en cuenta que, para entonces, la recesión económica se encontraba en cierto apogeo. De esta forma, se cuenta con tres periodos anuales analizados que suponen ser un "antes, durante y después" con respecto a la crisis económica. Por tanto, no se analiza una tendencia sino, más bien, se comparan tres momentos. Los datos metodológicos relativos a esta base de datos se pueden obtener remitiéndose al enlace que aparece en las referencias bibliográficas (Instituto Nacional de Estadística 2018).

Con respecto a la selección de edad, se consideran jóvenes a aquellos individuos de 16 a 34 años, ya que los 16 años es la edad mínima legal para que un joven pueda trabajar y emanciparse. Además, en esta investigación se considera, teniendo en cuenta la literatura existente, abordar el análisis del fenómeno emancipatorio hasta los 35 años como límite idóneo para contemplar el retraso de la marcha del hogar familiar. Sin embargo, se ha llevado a cabo una selección de casos que tomó como muestra a los jóvenes de 25 a 34 años, los cuales cumplían la característica de ser sustentadores principales. Cabe mencionar que se ha seleccionado ese intervalo tras una revisión exploratoria sobre la edad de emancipación de los jóvenes, en la que se ha comprobado que esta se sitúa más en este intervalo que en el de 16 a 24.

Respecto a la muestra seleccionada para la investigación, en la tabla 2 se observan los porcentajes de jóvenes entre 25 y 34 años que son, por un lado, sustentadores principales del hogar y, por otro, hijos o hijas de los sustentadores principales del hogar. Se observa que, en los tres años analizados, son más los jóvenes de 25 a 34 años (alrededor de un $60 \%$ ) que tienen como sostén familiar o persona de referencia económica a uno de sus progenitores y que, por tanto, no están emancipados. En la tabla 3 se puede observar una descripción de la muestra más detallada.

Tabla 2.

Muestra seleccionada para el análisis

\begin{tabular}{|c|c|c|c|}
\hline & EPF 2006 & EPF 2011 & EPF 2015 \\
\hline Jóvenes de 25 a 34 años & $\begin{array}{c}100 \% \\
5340\end{array}$ & $\begin{array}{c}100 \% \\
4925\end{array}$ & $\begin{array}{c}100 \% \\
3827\end{array}$ \\
\hline Sustentadores principales de 25 a 34 años & $\begin{array}{c}39,8 \% \\
2125 \\
\end{array}$ & $\begin{array}{c}43,5 \% \\
2144\end{array}$ & $\begin{array}{c}37,9 \% \\
1452 \\
\end{array}$ \\
\hline $\begin{array}{l}\text { Hijos o hijas de los sustentadores principa- } \\
\text { les de } 25 \text { a } 34 \text { años }\end{array}$ & $\begin{array}{c}60,2 \% \\
3215\end{array}$ & $\begin{array}{c}56,5 \% \\
2781\end{array}$ & $\begin{array}{c}62,1 \% \\
2445\end{array}$ \\
\hline
\end{tabular}

Fuente: Elaboración propia a partir de la Encuesta de Presupuestos Familiares (2006, 2011 y 2015). 
El uso del análisis descriptivo ha permitido observar las frecuencias de las diversas variables de interés en esta investigación ${ }^{1}$. Por otro lado, para obtener los resultados se han llevado a cabo diversos modelos de regresión logística binaria en función del año de realización de la Encuesta de Presupuestos Familiares. Con ello se busca llevar a cabo una comparación y un análisis que permitirá aceptar o refutar las hipótesis planteadas.

Se pretende llevar a cabo una comparación entre los datos de 2006, 2011 y 2015. Ambas bases de datos se han realizado bajo un mismo diseño muestral y tienen similares características. Sin embargo, de esta forma los modelos no son directamente comparables. Por ello se ha llevado a cabo el análisis de los efectos marginales medios, cuyos valores advierten en qué medida una determinada variable independiente incrementa o aminora la probabilidad de ser sustentador principal del hogar o ser hijo o hija de este; es decir, una aproximación porcentual al hecho de estar o no emancipado. Así, la diferencia que deriva de la comparación de ambas hipotéticas submuestras (sustentadores principales e hijos o hijas de sustentadores principales) resulta ser el efecto marginal medio de la variable analizada.

\section{Variables}

Como variable dependiente del modelo se ha tomado la "relación con el sustentador principal", es decir, "aquel miembro del hogar de 16 o más años cuya aportación periódica (no ocasional) al presupuesto común esté destinada a atender los gastos del hogar en mayor grado que las aportaciones de cada uno de los miembros restantes" (INE 2006; 2011; 2015). La utilización de esta variable ha sido considerada como una buena forma de aproximación al estudio de la emancipación juvenil por dos motivos.

En primer lugar, por el hecho de escoger el sustentador principal no se considera a todos los jóvenes emancipados, ya que puede una pareja emanciparse y solo uno de ellos tendrá dicha consideración. Sin embargo, un joven sustentador principal tiene una gran probabilidad de residir en un hogar propio, excepto en algún caso concreto en el que siga residiendo con sus progenitores o con alguno de ellos, a pesar de obtener más ingresos que estos.

En segundo lugar, se contempla de forma implícita el nivel de ingresos, variable también adecuada para conocer la autosuficiencia económica $y$, consecuentemente, residencial de los jóvenes, por lo que la utilización del sustentador principal como variable dependiente resulta ser bastante completa para el análisis.

Esta variable, en el modelo planteado, toma valor 1 si es sustentador principal y 0 si es hijo o hija del sustentador principal.
Como variables independientes del modelo de la investigación se han tomado las siguientes ${ }^{2}$ :

- El sexo.

- El tramo de edad, teniendo en cuenta que el valor 1 son los jóvenes que tienen entre 30 y 34 años, mientras que el valor 0 lo conforman aquellos que tienen de 25 a 29 años.

- El nivel de estudios, variable que toma el valor 1 en el caso de "estudios superiores", en la que se incluye la formación universitaria, postgrado y doctorado. Por otra parte, el valor 0 indica haber finalizado máximo hasta la ESO; es decir, se ramifica en las siguientes categorías: "sin estudios", "estudios primarios" y "estudios secundarios".

- El nivel de ingresos, cuyas categorías se agrupan en dos valores. Por una parte, el valor 1 lo adquieren aquellos jóvenes que ingresan entre 1000 y $2000 €$ al mes, mientras que el valor 0 lo obtienen los jóvenes que perciben $1000 €$ o menos mensualmente.

- La situación de actividad, variable que adquiere valor 1 cuando se trata de jóvenes con trabajo, mientras que el valor 0 reúne a aquellos que se encuentran parados, o bien su situación principal es la de estudiante.

\section{Hipótesis}

Las hipótesis desde las que se parte en este estudio se proponen siguiendo una serie de cuestiones reflejadas en la revisión bibliográfica anterior, las cuales se detallan a continuación:

- Hipótesis 1. Existen diferencias entre las variables que influyen en los perfiles de los jóvenes sustentadores principales o hijos o hijas de sustentadores principales de los años 2006, 2011 y 2015. Estas disimilitudes se deben al momento de la recesión económica en el que se encuentra cada una de esas fechas. Por un lado, 2011 se halla en plena crisis económica, mientras que 2006 es un año en el que habitaba la prosperidad y en 2015 ya existen claros síntomas de recuperación económica. Por tanto, concretamente existen más divergencias entre 2006 y las otras dos fechas; sin embargo, entre 2011 y 2015 no se esperan diferencias significativas.

- Hipótesis 1.1. En segundo lugar, el nivel de estudios en 2006 y en 2011 no influye en la probabilidad de que un joven sea sustentador principal; al contrario que en 2015 , en que esta probabilidad sí se ve afectada positivamente. La comparación entre fechas respecto a esta variable es muy relevante, puesto que, en los últimos años, entre otras cuestiones debido a la crisis económica, se ha producido una prolongación en el periodo 
formativo de los jóvenes, trayendo consigo consecuencias como el retraso de la incorporación al mercado laboral, lo que dificulta la estabilidad económica de aquellos. Por tanto, esta circunstancia conlleva que, al alargar la etapa formativa, un nivel de estudios superior estará claramente relacionado con los jóvenes que son hijos o hijas del sustentador principal del hogar y, por tanto, no están emancipados.

- Hipótesis 1.2. Con respecto al nivel de ingresos, unas retribuciones más altas implican una mayor probabilidad de que un joven sea sustentador principal del hogar y que, por tanto, esté emancipado. En cambio, un nivel de ingresos inferior a $1000 €$ mensuales se aproxima más a los jóvenes que son hijos o hijas del sustentador principal del hogar y que, por ende, viven con sus progenitores o con alguno de ellos.

- Hipótesis 1.3. Al tratar la situación de actividad, se considera que los jóvenes con más trabajo tienen más probabilidad de ser sustentadores principales de su hogar (de estar emancipados), mientras que los que se encuentran en situación de desempleo o están estudiando se aproximan más a la condición de hijo o hija del sustentador principal (no estar emancipados), indistintamente del año considerado.

- Hipótesis 1.4. Al tratar el tipo de contrato de los sustentadores principales del hogar en 2006 (prosperidad económica), 2011 (durante la crisis económica) y en 2015 (finalizada la crisis), tras las reformas y medidas contempladas en la tabla 1, se espera que existan prácticamente los mismos contratos indefinidos en 2011 y 2015 que en 2006. El abaratamiento del despido, entre otras cuestiones, conlleva que se siga contratando de forma indefinida, sin que esto impida despedir fácilmente. Por tanto, a pesar de que los contratos indefinidos no hayan disminuido, sí lo ha hecho la calidad de los mismos; la tenencia de un contrato indefinido ya no proporciona la misma estabilidad.

\section{RESULTADOS Y DISCUSIÓN}

En la tabla 3 se expone una descripción detallada de las variables anteriormente explicadas y utilizadas en el modelo. A grandes rasgos, se observan varias cuestiones. En primer lugar, con respecto al sexo, son más los varones sustentadores principales del hogar con respecto a las mujeres; sin embargo, la diferencia es menor conforme avanza el año de la muestra. Por tanto, se intuye que dichas disimilitudes por sexo se están atenuando con el paso de los años, aunque sigue siendo el varón en mayor medida el "cabeza de familia". Por otra parte, se observa claramente que el tramo de edad superior (30-34 años) se corresponde en gran medida a los jóvenes sustentadores principales del hogar, en detrimento del primer tramo de edad
(25-29 años). Además, los datos muestran que, con el paso del tiempo, es menos común ser sustentador principal dentro del primer tramo de edad, mientras que ocurre lo contrario con el segundo.

A continuación, con respecto al nivel de estudios, en general se observa que son más los jóvenes que tienen un nivel de estudios no superior a la ESO. Sin embargo, los porcentajes de este nivel de estudios son más altos cuando se trata de los jóvenes sustentadores principales del hogar, mientras que no existe tanta diferencia en el caso de los estudios superiores. Por su parte, en la siguiente tabla se aprecia que un nivel de ingresos más alto (1000 €-2000 $€$ mensuales) recibe un alto porcentaje de jóvenes sustentadores principales, mientras que un nivel de ingresos más bajo (menos de $1000 €$ ) está altamente relacionado con aquellos jóvenes que son hijos o hijas del sustentador principal del hogar. En ambas variables no se observan diferencias significativas en función del año.

Los resultados de las estimaciones para 2006, 2011 y 2015 se exponen en la tabla 4. Estas permiten contrastar que la bondad de ajuste es apta para obtener conclusiones, ya que el test Chi-cuadrado, que comprueba si los modelos son globalmente significativos, arroja un $p$-valor menor del $0,1 \%$ en ambos. Así mismo, los efectos marginales que se muestran en la tabla 4, en porcentajes, permiten conocer los factores individuales de los sujetos analizados que aumentan o disminuyen la probabilidad de que un joven sea sustentador principal, así como también la exactitud porcentual de esa aproximación o lejanía con respecto a la variable dependiente.

A través de esta forma de conocer los resultados marginales, se presupone una combinación de dos tipos de poblaciones hipotéticas que tienen exactamente los mismos valores en las otras variables independientes del modelo. Como dice Williams (2012), la lógica es similar a la de un estudio de correspondencia, en que los sujetos tienen valores idénticos en cada variable independiente, excepto en una. Sin embargo, el mayor problema de dicha estimación (a través de valores promedios) es que dichas cifras pueden subestimar las diferencias en los efectos entre los casos. Por ejemplo, el efecto que variables como el sexo tienen sobre la probabilidad de ser sustentador principal varía según las características de la persona; las diferencias podrían ser mucho mayores para las personas con, por ejemplo, estudios superiores que para los que tienen estudios primarios. Esta problemática, además de otras, lleva a que muchos investigadores utilicen otros comandos que implican otras funciones para estimar los efectos marginales. Existe una función que muestra cómo los efectos de las variables varían según otras características del individuo, por lo que se ajusta mejor a la realidad. No obstante, en modelos con muchas variables, como es el caso, resulta bastante engorroso llevar a cabo dicho análisis (Williams 2012). 
Tabla 3.

Descripción de la muestra seleccionada

\begin{tabular}{|c|c|c|c|c|c|c|c|}
\hline \multirow[b]{2}{*}{ Variables } & & \multicolumn{2}{|c|}{$\begin{array}{l}\text { Encuesta presupuestos } \\
\text { familiares } 2006\end{array}$} & \multicolumn{2}{|c|}{$\begin{array}{l}\text { Encuesta presupuestos } \\
\text { familiares } 2011\end{array}$} & \multicolumn{2}{|c|}{$\begin{array}{l}\text { Encuesta presupuestos } \\
\text { familiares } 2015\end{array}$} \\
\hline & & $\begin{array}{l}\text { Sustentador } \\
\text { principal }\end{array}$ & $\begin{array}{l}\text { Hijo/a del } \\
\text { sustentador } \\
\text { principal }\end{array}$ & $\begin{array}{l}\text { Sustentador } \\
\text { principal }\end{array}$ & $\begin{array}{l}\text { Hijo/a del } \\
\text { sustentador } \\
\text { principal }\end{array}$ & $\begin{array}{l}\text { Sustentador } \\
\text { principal }\end{array}$ & $\begin{array}{c}\text { Hijo/a del } \\
\text { sustentador } \\
\text { principal }\end{array}$ \\
\hline \multirow{2}{*}{\multicolumn{2}{|c|}{ Sustentador principal }} & 2125 & 3215 & 2144 & 2781 & 1452 & 2445 \\
\hline & & $39,79 \%$ & $60,21 \%$ & $43,53 \%$ & $56,47 \%$ & $37,94 \%$ & $62,06 \%$ \\
\hline \multirow{4}{*}{ Sexo } & \multirow{2}{*}{ Hombre } & 1689 & 1825 & 1451 & 1594 & 916 & 1433 \\
\hline & & $79,48 \%$ & $36,77 \%$ & $67,68 \%$ & $57,32 \%$ & $63,09 \%$ & $58,61 \%$ \\
\hline & \multirow{2}{*}{ Mujer } & 436 & 1390 & 693 & 1187 & 536 & 1012 \\
\hline & & $20,52 \%$ & $43,23 \%$ & $32,32 \%$ & $42,68 \%$ & $36,91 \%$ & $41,39 \%$ \\
\hline \multirow{4}{*}{ Edad } & \multirow{2}{*}{ 24-29 años } & 457 & 1845 & 444 & 1515 & 238 & 1397 \\
\hline & & $21,51 \%$ & $57,39 \%$ & $20,71 \%$ & $55,27 \%$ & $16,39 \%$ & $57,13 \%$ \\
\hline & \multirow{2}{*}{ 30-35 años } & 1668 & 1370 & 1700 & 1266 & 1214 & 1048 \\
\hline & & $78,49 \%$ & $42,61 \%$ & $79,29 \%$ & $45,52 \%$ & $83,61 \%$ & $42,87 \%$ \\
\hline \multirow{4}{*}{$\begin{array}{l}\text { Nivel de } \\
\text { estudios }\end{array}$} & \multirow{2}{*}{ ESO o menor nivel } & 1337 & 1668 & 1285 & 1536 & 839 & 1298 \\
\hline & & $62,92 \%$ & $51,88 \%$ & $59,93 \%$ & $55,27 \%$ & $57,78 \%$ & $53,11 \%$ \\
\hline & \multirow{2}{*}{ Estudios superiores } & 788 & 1546 & 859 & 1,243 & 613 & 1146 \\
\hline & & $37,08 \%$ & $48,12 \%$ & $40,07 \%$ & $44,73 \%$ & $42,22 \%$ & $46,89 \%$ \\
\hline \multirow{4}{*}{$\begin{array}{l}\text { Intervalo de } \\
\text { ingresos }\end{array}$} & \multirow{2}{*}{$<1.000 €$ mensuales } & 637 & 1156 & 636 & 919 & 482 & 785 \\
\hline & & $32,92 \%$ & $64,76 \%$ & $32,35 \%$ & $60,54 \%$ & $35,70 \%$ & $67,79 \%$ \\
\hline & \multirow{2}{*}{$\begin{array}{l}\text { De } 1.000 \text { a } 2.000 € \\
\text { mensuales }\end{array}$} & 1298 & 629 & 1330 & 599 & 868 & 373 \\
\hline & & $67,08 \%$ & $35,24 \%$ & $67,65 \%$ & $39,46 \%$ & $64,30 \%$ & $32,21 \%$ \\
\hline \multirow{4}{*}{$\begin{array}{l}\text { Situación de } \\
\text { actividad }\end{array}$} & \multirow{2}{*}{ Con trabajo } & 2019 & 2349 & 1852 & 1599 & 1285 & 1233 \\
\hline & & $95,91 \%$ & $75,70 \%$ & $87,03 \%$ & $59,75 \%$ & $89,17 \%$ & $51,95 \%$ \\
\hline & \multirow{2}{*}{$\begin{array}{l}\text { Parados o } \\
\text { estudiantes }\end{array}$} & 86 & 754 & 276 & 1077 & 156 & 1140 \\
\hline & & $4,09 \%$ & $24,30 \%$ & $12,97 \%$ & $40,25 \%$ & $10,83 \%$ & $48,05 \%$ \\
\hline
\end{tabular}

Fuente: Elaboración propia a partir de la Encuesta de Presupuestos Familiares (2006, 2011 y 2015).

Seguidamente, se procede a comentar los resultados obtenidos en función de las hipótesis. Como se puede observar en la tabla 4, las relaciones entre variables independientes y la variable dependiente tienen prácticamente la misma dirección, por lo que no podemos aceptar la hipótesis 1 . Sin embargo, sí es cierto que, dependiendo de la fecha analizada, las relaciones entre variables, a pesar de no cambiar de dirección, sí varían su fuerza o peso, por lo que se pueden trazar algunas tendencias.

En primer lugar, en la tabla 4 se observa que la variable "sexo" tiene una relación estadísticamente significativa con el hecho de ser sustentador principal o bien ser hijo o hija del mismo en 2006, siendo el varón el que tiene mayor probabilidad (un varón tiene un $16,9 \%$ más de probabilidades de ser sustentador principal del hogar que una mujer). Sin embargo, a pesar de que en 2011 y 2015 la relación no es significativa, y por ello no se pueden extraer ciertas conclusiones, cabe puntualizar un pequeño cambio de tendencia. Mientras que en 2011 el varón sigue teniendo una mayor probabilidad de ser sustentador principal con respecto a la mujer, esta es menor que en 2006, y en 2015 el símbolo negativo indica que esa tendencia puede estar revertiéndose, siendo la mujer la que cuente con mayor probabilidad.

En definitiva, como indica Hernández (2012b: 82), las diferencias de género en el ámbito laboral aún persisten en España. Por ejemplo, en el caso de las mujeres, la temporalidad de sus empleos es más frecuente que en el caso de los varones (Hernández y López 2015) y su incorporación al mercado laboral, más difícil (Pérez-Baleón 2012). Ambas cuestiones dificultan la emancipación de las mujeres en comparación con los varones. Sin embargo, estas utilizan "el método tradicional" de emanciparse a través de la unión en pareja a una edad más temprana que los varones (Moreno 2012b). Tal y como se observa en la tabla 4, son muchos más los varones sustentadores principales del hogar que las mujeres. Sin embargo, esto no quiere decir que las mujeres se emancipen con menor frecuencia. 
Al detenernos en el gráfico 2, se observa que las parejas del sustentador principal del hogar son, en gran medida, mujeres, así como que también vuelve a aparecer la evidencia de que la mayoría de los sustentadores principales del hogar son varones. Por tanto, se evidencia este hecho; la unión en pareja es un gran detonante para emanciparse en el caso de las mujeres y en relación con los varones. Sin embargo, cabe puntualizar que, al comparar las tres fechas consideradas, esta tendencia se está invirtiendo. El porcentaje de mujeres que son parejas del sustentador principal está descendiendo (de un $90,7 \%$ en 2006 ha pasado a un $80,7 \%$ en 2015), mientras que el de mujeres sustentadoras principales del hogar ha aumentado (de un $20,5 \%$ en 2006 ha pasado a un $36,9 \%$ en 2015). De la misma forma, el porcentaje de varones sustentadores principales del hogar ha descendido; mientras que en 2006 un $79,5 \%$ de los sustentadores principales eran varones, en 2015 esta cifra ha bajado a un 63,1\%.

En segundo lugar, con respecto al tramo de edad analizado, se observa en la tabla 4 que, en términos generales, un joven de 30 a 34 años tiene mucha más probabilidad de ser el sustentador principal del hogar y de estar emancipado que uno de 25 a 29 años. Además, con el paso de los años dicha probabilidad aumenta: en 2006, un joven de 30 a 34 años tenía un $32,9 \%$ de probabilidad de ser el sustentador principal del hogar; en 2011 el hecho de tener entre 30 y 34 años implicaba una probabilidad del $31,3 \%$ más de ser sustentador principal del hogar que de ser hijo o hija del mismo (estar o no emancipado), mientras que en 2015 la probabilidad aumenta hasta el 41,8 \%.
La hipótesis 1.1, así mismo, indica que el nivel de estudios en 2006 y 2011 no influía en la probabilidad de que un joven sea sustentador principal, al contrario que en 2015, año en que esta probabilidad sí se ve afectada positivamente. Se comprueba en la tabla 4 que la categoría "estudios superiores" es significativa en las tres fechas, por lo que no se acepta la primera parte de la hipótesis. Sin embargo, cabe resaltar que los jóvenes con estudios superiores tienen menos probabilidad de ser sustentadores principales del hogar que aquellos jóvenes cuyo máximo nivel de estudios no supera la ESO; en 2006, un joven con estudios superiores tiene un $13,2 \%$ menos de probabilidad de ser el sustentador principal del hogar; en 2011 dicha probabilidad es de un $15,2 \%$ menos, mientras que en 2015 es de un $16 \%$. Por tanto, la segunda parte de la hipótesis se acepta, la cual incidía en que un nivel de estudios superior estará claramente relacionado con los jóvenes que son hijos o hijas del sustentador principal del hogar y, por tanto, no están emancipados.

Así, los estudios superiores no están relacionados con el hecho de ser sustentador principal, sino más bien al contrario, lo que se explica por el tramo de edad escogido para el análisis (24-35 años), pues, en muchas ocasiones, los jóvenes que están llevando a cabo dichos estudios todavía están formándose. Se percibe que la prolongación de la etapa formativa y, por tanto, el nivel de estudios superiores, incide en el retraso de la emancipación de los jóvenes. Esto puede deberse, como ya se apuntaba, a la inestabilidad laboral y económica en la que se encuentran los jóvenes en la actualidad, como también señalan algunos autores (Miret 2006; Ortega y Martín 2012). Por ello, a

Gráfico 2.

Relación con el sustentador principal por sexo y año (jóvenes de 25 a 34 años)

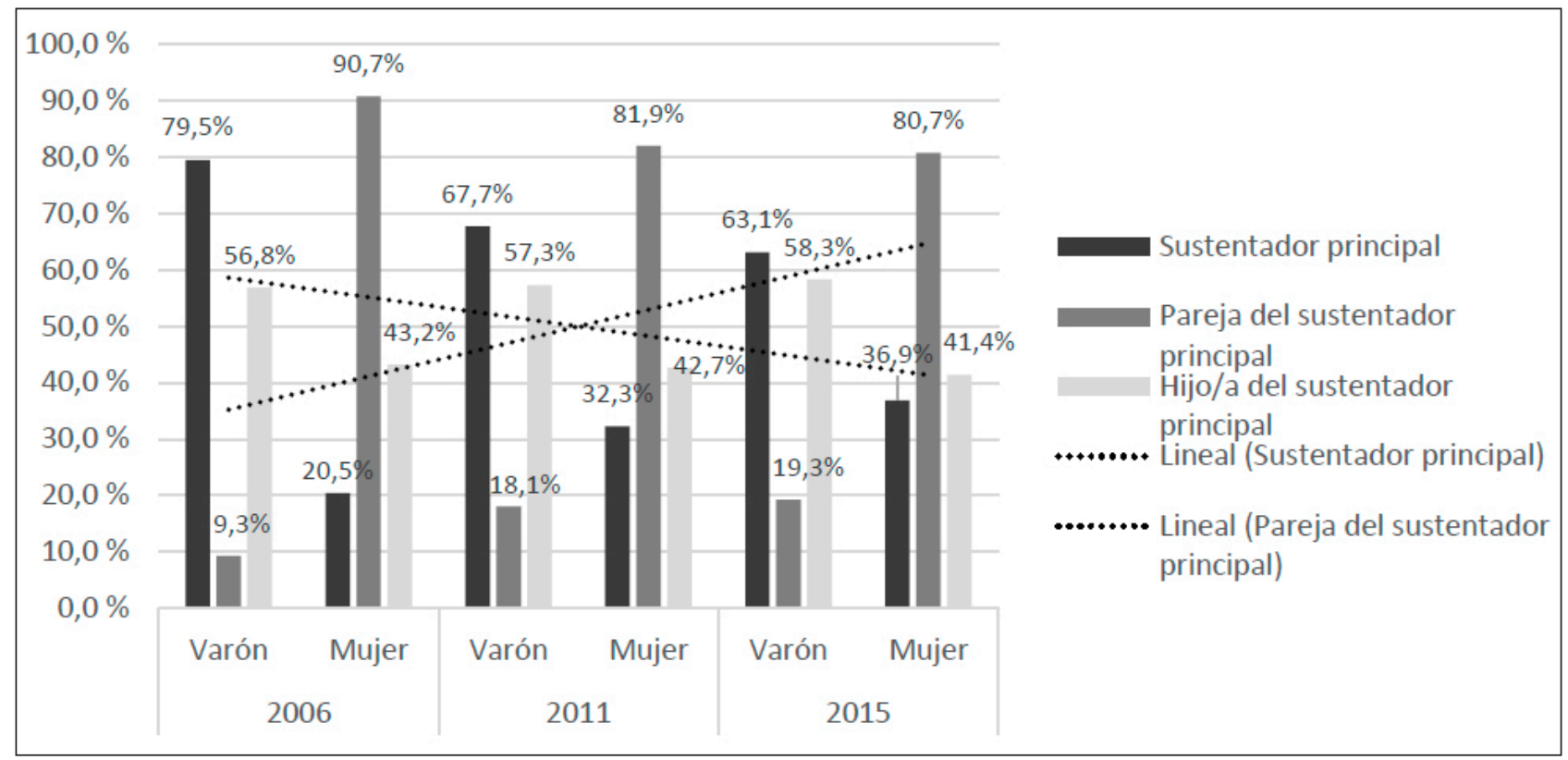

Fuente: elaboración propia a partir de la Encuesta de Presupuestos Familiares (2006, 2011 y 2015). 
la luz de los resultados, podemos conjeturar que el incumplimiento de las expectativas de los jóvenes, en lo que respecta a las condiciones laborales y a los salarios, da lugar a que estos opten por seguir formándose. De este modo, pretenden conseguir un trabajo que cumpla sus expectativas Una vez que lo consiguen, aumenta la probabilidad de que formen un hogar propio; mientras tanto, se inclinan por seguir conviviendo con sus progenitores. Esto les permite ahorrar dinero y continuar con su formación académica, a la vez que mantienen su nivel de bienestar y de consumo.

Se observa en la tabla 4 que los jóvenes con mayores ingresos (entre $1000 €$ y $2000 €$ mensuales) se aproximan más al hecho de ser sustentadores principales del hogar. Esta circunstancia ocurre en las tres fechas analizadas, aunque es cierto que cada año se va incrementando dicha probabilidad; mientras que en 2006 era de un $30 \%$, en 2011 pasó a ser un 31,5 \% y en 2015 un $36 \%$ más probable de que un joven que percibía entre $1000 €$ y $2000 €$ mensuales fuera sustentador principal del hogar y, por ello, estuviera emancipado, que un joven que obtenía una retribución menor de $1000 €$. Por tanto, parece que, conforme pasan los años, más determinante resulta el hecho de percibir una cantidad de ingresos alta para ser sustentador principal del hogar y, de esta forma, estar emancipado. De ahí podemos deducir que percibir ingresos inferiores a los $1000 €$ mensuales (es decir, ser mileurista), cada vez aleja más la posibilidad de ser sustentador principal del hogar y, por tanto, de emanciparse. Así, se acepta la hipótesis 1.2, puesto que la misma establecía esta misma relación, así como también que no existían diferencias significativas en función del año.

Por último, al tratar la situación de actividad destaca que los jóvenes con trabajo se aproximan más al hecho de no ser sustentadores principales del hogar, al contrario de lo que se sugería en la hipótesis 1.3, por lo que se rechaza la misma. Se observa en las tres fechas analizadas que, aunque en 2006 la relación entre ambas variables no es estadísticamente significativa, los jóvenes con trabajo tienen menos probabilidad de ser "cabezas de familia". ¿A qué puede deberse? En primer lugar, hay que señalar que muchos jóvenes con trabajo no son el sustentador principal del hogar, sobre todo en los casos en los que más de un miembro del hogar percibe ingresos. Lo que ocurre en muchos casos es que existe al menos un miembro percibiendo más ingresos que el joven en cuestión; por ejemplo, en los casos en los que los jóvenes son hijos o hijas del sustentador principal y ellos también trabajan, aunque no sean el principal sostén económico ni estén emancipados. Así, y en la línea de lo ya comentado con respecto al nivel de ingresos (ser mileurista no garantiza ser sustentador principal del hogar), tener trabajo no tiene por qué favorecer el hecho de que un joven sea sustentador principal, mientras que ser estudiante o estar en paro sí favorece el hecho de no serlo (a penas existen sustentadores principales dentro de esta condición).
Con respecto al tipo de contrato de los jóvenes sustentadores principales, en primer lugar, se observa que, a mayor edad, mejores condiciones tienen los jóvenes en lo que respecta al tipo de contrato, tal y como refleja la tabla 5. Mientras que en el grupo de edad de sustentadores principales de 25 a 29 años un 59,54\% de estos tiene un contrato indefinido, en el caso de los que tienen entre 30 y 34 años se trata de un 69,72\%. En el caso del contrato eventual, el porcentaje es mayor en los jóvenes de menor edad (el 37,73 \% son jóvenes de 25 a 29 años, mientras que un 28,52 \% pertenece a los que tienen entre 30 y 34 años) $)^{3}$.

Tratando el sexo, en la tabla 5 se puede observar como el varón goza de mejores condiciones en lo que respecta al tipo de contrato que la mujer. Concretamente, tienen en mayor medida contratos indefinidos $(68,17 \%$ frente a un $60,41 \%$ de las mujeres), mientras que son las mujeres las que, aunque no de forma sustancial, trabajan con contratos de peores condiciones; en el caso del contrato eventual, un $32,76 \%$ son mujeres, mientras que un $30,56 \%$ son varones. Finalmente, un $3,83 \%$ de mujeres trabajan sin contrato, mientras que son solo un $1,27 \%$ de los varones lo hacen.

Si observamos el nivel de ingresos, existe una alta correspondencia entre el nivel de ingresos y el tipo de contrato. En el caso de contratos indefinidos, son muchos más los jóvenes que perciben entre $1000 €$ y 2000 $€(44,34 \%)$ o más de $2000 €(47,37 \%)$ que los que perciben menos de $1000 €(8,29 \%)$. Aquellos jóvenes que son sustentadores principales con un contrato eventual, perciben, en su mayoría, entre $1000 €$ y 2000 $€(44,35 \%)$, aunque el porcentaje de los que ganan menos de $1000 €$ es mayor, y menor la categoría superior. Finalmente, con respecto a aquellos jóvenes sustentadores principales que no tienen contrato, la mayoría de estos perciben menos de $1000 €$ mensuales $(59,81 \%)$.

Finalmente, a fin de contrastar la hipótesis 1.4, la cual considera que, tras las reformas y medidas contempladas en la tabla 1, se espera que existan prácticamente los mismos contratos indefinidos en 2011 y 2015 que en 2006. Tal y como se observa en la tabla 5 , el descenso en el número de contratos indefinidos es insignificante, siendo de media en las tres fechas un $66 \%$ aproximadamente. Por otra parte, es de un $31 \%$ el porcentaje de jóvenes que, en los tres años considerados, tienen un contrato eventual, mientras que un $2 \%$ trabaja sin contrato. Por tanto, puesto que no existe apenas diferencias entre fechas, se acepta la hipótesis 1.4. Así, se observa que el número de contratos indefinidos no ha disminuido; únicamente lo ha hecho la calidad de los mismos. Esto explica que las condiciones laborales actuales de los jóvenes no proporcionen una estabilidad económica suficiente como para que puedan abandonar el hogar familiar (Gentile, 2016). En otras etapas, un contrato fijo proporcionaba estabilidad económica y laboral (Hidalgo y Pérez, 2004). En la actualidad no es así, debido a la precarización de las condiciones laborales de las que se trata en el marco analítico. 


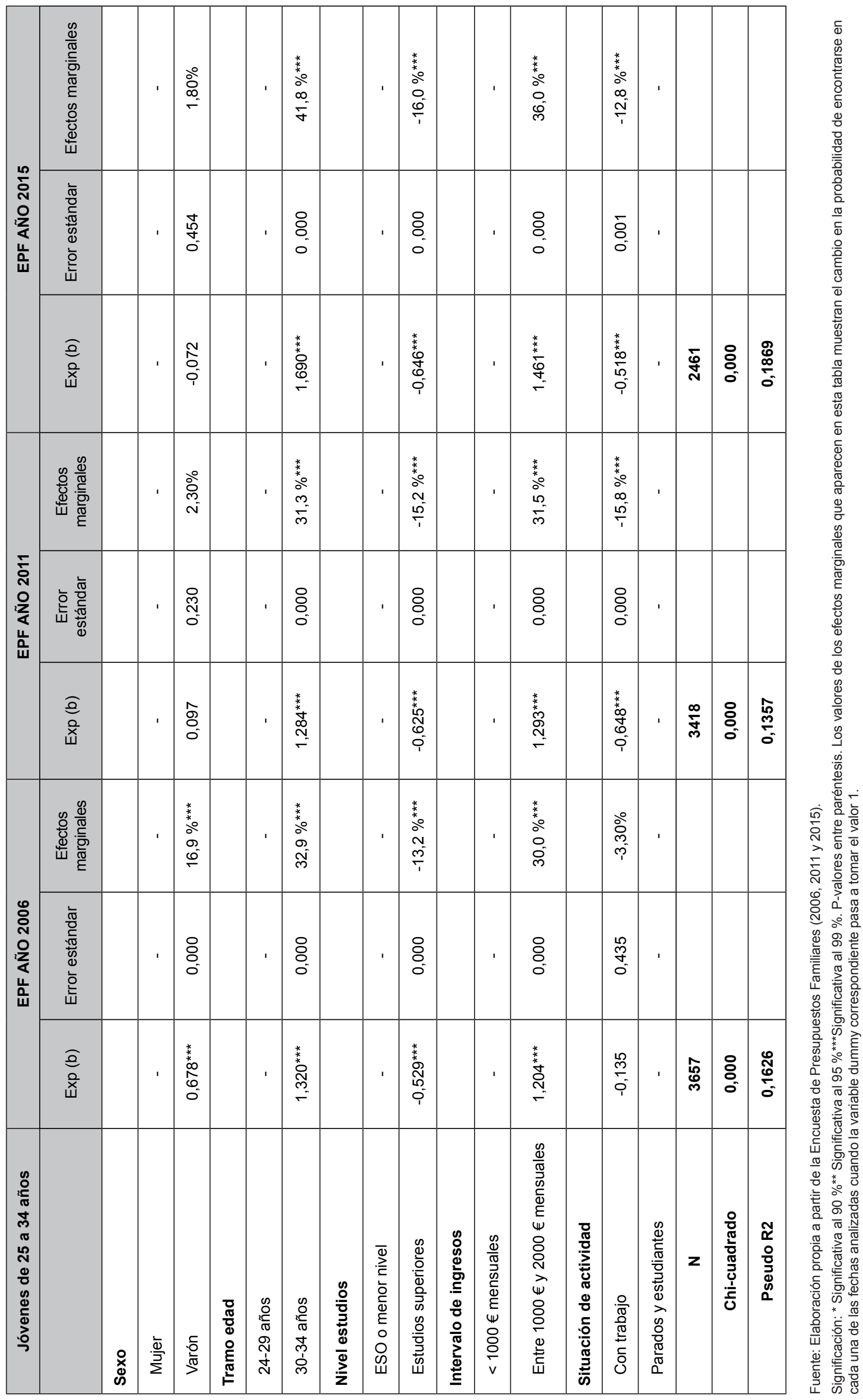


Tabla 5.

Tipo de contrato en función de una serie de variables independientes en jóvenes sustentadores principales de 25 a 34 años

\begin{tabular}{|c|c|c|c|c|}
\hline & & \multicolumn{3}{|c|}{ TIPO DE CONTRATO } \\
\hline & & Indefinido & Eventual & Sin contrato \\
\hline \multirow{2}{*}{ SEXO } & Varón & $\begin{array}{c}68,17 \% \\
2367\end{array}$ & $\begin{array}{c}30,56 \% \\
1061\end{array}$ & $\begin{array}{c}1,27 \% \\
44\end{array}$ \\
\hline & Mujer & $\begin{array}{c}60,41 \% \\
962\end{array}$ & $\begin{array}{c}32,76 \% \\
497\end{array}$ & $\begin{array}{c}3,83 \% \\
58\end{array}$ \\
\hline \multirow{2}{*}{ TRAMO DE EDAD } & $25-29$ años & $\begin{array}{c}59,54 \% \\
873\end{array}$ & $\begin{array}{c}37,73 \% \\
553\end{array}$ & $\begin{array}{c}2,73 \% \\
40\end{array}$ \\
\hline & 30-34 años & $\begin{array}{c}69,72 \% \\
2456\end{array}$ & $\begin{array}{c}28,52 \% \\
1005\end{array}$ & $\begin{array}{c}1,76 \% \\
62\end{array}$ \\
\hline \multirow{3}{*}{$\begin{array}{l}\text { NIVEL DE INGRESOS } \\
\text { MENSUALES }\end{array}$} & Menos de $1000 €$ & $\begin{array}{c}8,29 \% \\
276\end{array}$ & $\begin{array}{c}32,67 \% \\
509\end{array}$ & $\begin{array}{c}59,81 \% \\
61\end{array}$ \\
\hline & De $1000 €$ a $2000 €$ & $\begin{array}{c}44,34 \% \\
1476\end{array}$ & $\begin{array}{c}44,35 \% \\
691\end{array}$ & $\begin{array}{c}32,35 \% \\
33\end{array}$ \\
\hline & Más de $2000 €$ & $\begin{array}{c}47,37 \% \\
1577\end{array}$ & $\begin{array}{c}22,98 \% \\
358\end{array}$ & $\begin{array}{c}7,84 \% \\
8\end{array}$ \\
\hline \multirow{3}{*}{ AÑO } & 2006 & $\begin{array}{c}66,72 \% \\
1205\end{array}$ & $\begin{array}{c}31,78 \% \\
574\end{array}$ & $\begin{array}{c}1,50 \% \\
27\end{array}$ \\
\hline & 2011 & $\begin{array}{c}66,33 \% \\
1261\end{array}$ & $\begin{array}{c}31,09 \% \\
591\end{array}$ & $\begin{array}{c}2,58 \% \\
49\end{array}$ \\
\hline & 2015 & $\begin{array}{c}67,32 \% \\
863\end{array}$ & $\begin{array}{c}30,66 \% \\
393\end{array}$ & $\begin{array}{c}2,02 \% \\
26\end{array}$ \\
\hline
\end{tabular}

Fuente: elaboración propia a partir de la Encuesta de Presupuestos Familiares (2006, 2011 y 2015).

\section{Conclusiones}

Este artículo versa acerca de la emancipación de los jóvenes a partir de su relación con el sustentador principal del hogar, analizando dos perfiles: los jóvenes sustentadores principales del hogar y los jóvenes hijos o hijas de los sustentadores principales del hogar. Una vez conocido el peso de ciertas variables socioeconómicas en la configuración de dichos perfiles, se ha comprobado en qué medida afecta la recesión económica al respecto, tomando como referencia tres fechas: 2006, 2011 y 2015.

Respecto a las hipótesis planteadas, la primera hacía referencia a la existencia de divergencias entre los perfiles de los jóvenes sustentadores principales entre los años 2006, 2011 y 2015, con algunos matices. A este respecto, se ha observado que las variables que influyen en la configuración de los perfiles de los jóvenes sustentadores principales no difieren considerablemente en función del año; sin embargo, sí podemos señalar ciertas tendencias.
En términos generales, cabe puntualizar que, a pesar de que no existen diferencias muy significativas en función del año analizado, los jóvenes cada vez se emancipan más tarde. La edad media para ser un joven sustentador principal del hogar ( $y$, en su caso, estar emancipado), ha aumentado desde los 30,65 años (2006) a los 30,72 en 2011 y, finalmente, a los 31,05 años en 2015. En épocas de crisis económicas, en las que también el mercado de trabajo se ve afectado, el nivel de incertidumbre aumenta. Este hecho explica que se produzca un retraso en la salida del hogar familiar. Seguir en el hogar de los progenitores, por un lado, permite a los jóvenes enfrentarse a las dificultades del mercado de trabajo y, por otro, les posibilita seguir formándose o mantenerse en la búsqueda un empleo que concuerde con sus capacidades y formación (López 2006; Vieira y Gamundi 2010). Otro hecho que llama la atención al respecto es que la mujer, a pesar de tener menos probabilidad que el varón de ser sustentadora principal del hogar, cuando lo es, lo es de forma más 
temprana que el varón. Así, mientras que la media, en 2006, en el caso del varón era de 30,78 , la de la mujer era de 30,52 años. Lo mismo ocurre en 2011 (31,03 años el varón y 30,42 la mujer) y en 2015 (31,22 años el varón y 30,76 la mujer). Además, se observa que dichas divergencias en función del sexo cada año son más amplias.

En cuanto a la hipótesis 1.1, tanto en 2006 como en 2011 y 2015, el nivel de estudios influye en la probabilidad de que un joven sea sustentador principal, por lo que no se acepta. Si bien cabe resaltar que el hecho de tener estudios superiores está relacionado con una menor probabilidad de ser sustentador principal del hogar $y$, por tanto, de estar emancipado; así, se acepta la segunda parte de la hipótesis. La prolongación de la etapa formativa derivada de la inestabilidad laboral y económica (precarización de las condiciones laborales, entre otras cuestiones) en la que se encuentran los jóvenes en la actualidad retrasa la transición de los jóvenes hacia la edad adulta. El hecho de que no se cumplan las expectativas de los jóvenes en lo que respecta a las condiciones laborales y los salarios da lugar a que los jóvenes opten por seguir formándose, a la espera de lograr un trabajo que coincida con sus intereses.

Por otra parte, se acepta la hipótesis 1.2, la cual señala que unos ingresos más altos implican una mayor probabilidad de que un joven sea sustentador principal del hogar, sin existir cambios considerables en función del año considerado. Sin embargo, sí aparece cierta tendencia, la cual muestra que, conforme pasan los años, más determinante resulta el hecho de percibir una cantidad de ingresos más alta (entre $1000 €$ y $2000 €$ ) en la cuestión de ser sustentador principal del hogar $y$, de esta forma, estar emancipado. Así, percibir ingresos inferiores a los $1000 €$ mensuales (ser mileurista), cada vez aleja más de la posibilidad de ser sustentador principal del hogar $y$, por tanto, de emanciparse.

Al tratar la situación de actividad, se rechaza la hipótesis 1.3, la cual indica que los jóvenes con trabajo se aproximan más al hecho de ser sustentadores principales del hogar. Lo que ocurre en muchos casos es que existe un miembro o más en el hogar percibiendo ingresos, además del joven en cuestión. Así, y en la línea de lo ya comentado con respecto al nivel de ingresos (ser mileurista no garantiza ser sustentador principal del hogar), tener trabajo no tiene por qué favorecer el hecho de que un joven sea sustentador principal, mientras que sí lo es el hecho de ser estudiante o estar en paro; existen muy pocos sustentadores principales dentro de esta condición.

Finalmente, la hipótesis 1.4 plantea que existen prácticamente los mismos contratos indefinidos en 2011 y en 2015 que en 2006, por lo que se acepta la hipótesis. El número de contratos indefinidos no han disminuido, únicamente lo ha hecho la calidad de los mismos. Así, tanto la inestabilidad laboral como las condiciones laborales de los jóvenes, lejos de proporcionar un equilibrio laboral y económico, no permiten que puedan abandonar el hogar familiar (Gentile 2016).

Se prevé que esta investigación pueda tener cierta repercusión, puesto que puede abrir ciertas líneas de intervención pública. Por ejemplo, se observa claramente que el contrato indefinido, tal y como está establecido, ya no aporta los beneficios que originaba antes. Por su parte y con respecto al nivel de ingresos, la condición de mileurista tampoco parece ser suficiente como para hacerse cargo de un hogar o vivir de forma independiente. Además, los datos ponen en duda, en cierta medida, la eficacia de las reformas que se han llevado a cabo en los últimos años (por ejemplo, la flexibilización y el abaratamiento del despido). Llama la atención que la evolución del Salario Mínimo Interprofesional sea positiva y, sin embargo, el salario medio de los jóvenes haya descendido en los últimos años. De ello podemos concluir que las condiciones de contratación se han visto deterioradas en este sector de la población. Estos resultados serán comparados con la situación experimentada por otros países de nuestro entorno como futura línea de investigación.

\section{AgRADECIMIENTOS Y FINANCIACIÓN}

Este artículo ha sido financiado por el Fondo Social Europeo en colaboración con la Junta de Andalucía y la Universidad de Granada, organismos a los que mostramos nuestra gratitud. Además, se ha contado con la generosa colaboración de Jorge Guardiola Wanden-Berghe, al que le agradecemos su paciencia y dedicación. Los posibles errores contenidos en este artículo son responsabilidad exclusiva de los autores.

\section{NOTAS}

[1] Para llevar a cabo la selección de las variables, nos remitimos a los factores destacados por Ahn y Sánchez-Marcos (2017) que influyen en la emancipación de los jóvenes.

[2] Se ha llevado a cabo una recodificación de la mayoría de las variables en dos categorías, con el fin de simplificar el análisis y, además, obtener resultados más contundentes.
[3] El tipo de contrato se subdivide en: 1) indefinido, contratos que no se extinguen mientras no se produzca un despido; 2) eventual o temporal, donde se incluyen los contratos definidos, y 3 ) sin contrato, lo que se refiere al caso en que no exista un contrato formal y firmado, incluso en trabajos regulares o de larga duración. 


\section{RefERENCIAS BibLIOGRÁficas}

Ahn, N. y V. Sánchez-Marcos. 2017. "Emancipation under the great recession in Spain". Review of Economics of the Household 15(2): 477-495. https://doi.org/10.1007/ s11150-015-9316-7

Arnett, J. J. 2014. Emerging adulthood: The winding road from the late teens through the twenties. New York: Oxford University Press. https://doi.org/10.1093/acprof:o so/9780199929382.003.0013

Aparicio-Fenoll, A. y V. Oppedisano, V. 2012. "Fostering the emancipation of young people: evidence from a Spanish rental subsidy". Working Papers 6651. Institute for the Study of Labor (IZA).

Ayuso, L. 2010. "Juventud y familia en los comienzos del siglo XXI”. Pp. 115-175 en Jóvenes españoles 2010. Madrid: Fundación Santa María.

Becker, S. O., S. Bentolila, A. Fernandes y A. Ichino. 2010. "Youth emancipation and perceived job insecurity of parents and children". Journal of Population Economics 23(3): 1047-1071. https://doi.org/10.1007/s00148-008-0224-5

Berngruber, A. 2015. "Generation boomerang in Germany? Returning to the parental home in young adulthood". Journal of Youth Studies 18(10): 1274-1290. https:// doi.org/10.1080/13676261.2015.1039969

Blossfeld, H. P., E. Klijzing, M. Mills y K. Kurz. 2006. Globalization, uncertainty and youth in society: The losers in a globalizing world. London: Routledge.

Bourdieu, P. 2000. Contrafuegos: reflexiones para servir a la resistencia contra la invasión neoliberal. Barcelona: Anagrama.

Burn, K. y C. Szoeke. (2016). "Boomerang families and failure-to-launch: Commentary on adult children living at home". Maturitas 83: 9-12. https://doi.org/10.1016/j. maturitas.2015.09.004

Ermisch, J. 1999. "Prices, parents, and young people's household formation". Journal of Urban Economics 45(1): 47-71. https://doi.org/10.1006/juec.1998.2083

Ermisch, J. y P. Di Salvo. 1997. "The economic determinants of young people's household formation”. Económica 64(256): 627-644. https://doi.org/10.1111/14680335.00103

Eurostat (2016). Youth unemployment by sex, age and educational attainment level. Consulta 10 de junio de 2017 (https://appsso.eurostat.ec.europa.eu/nui/show. do?dataset=yth_empl_090\&lang=en).

Fernández, J. A. (1997). "Youth residential independence and autonomy: A comparative study". Journal of family issues 18(6): 576-607. https://doi. org/10.1177/019251397018006002

García, M. y R. Merino. 2006. "Transición a la vida adulta: nuevas y viejas desigualdades en función del género". Revista Española Internacional de Sociología 113: 155-162.

García-Montalvo, J. (2009). "La inserción laboral de los universitarios y el fenómeno de la sobrecualificación en España". Papeles de economía española 119: 172-187.

Garrido, L., M. Requena y J. Rivière. 1995. La emancipación de los jóvenes en España. Madrid: Ministerio de Trabajo e inmigración, INJUVE.

Gee, E., B. Mitchell y A. Wister. 1995. "Returning to the parental "nest": exploring a changing canadian life course".
Canadian Studies in Population 22(2): 121-144. https:// doi.org/10.25336/P6WK5T

Gentile, A. 2010. "De vuelta al nido en tiempos de crisis. Los boomerang kids españoles". Revista de estudios de juventud 90: 181-203.

Gentile, A. 2015. "Jóvenes titulados superiores en la encrucijada de la crisis". Recerca, Revista de Pensament $i$ Anàlisi 16: 35-58.

Gentile, A. 2016. "La emancipación juvenil en tiempos de crisis: un diagnóstico para impulsar la inserción laboral y la transición residencial". Metamorfosis 119-124.

Giannelli, G. y C. Monfardini. 2003. "Joint decisions on household membership and human capital accumulation of youths. The role of expected earnings and local markets". Journal of Population Economics 16(2): 265285. https://doi.org/10.1007/s001480200119

Hernández, J. 2012a. “¿Flexibilidad laboral, flexiseguridad o flexplotación?”. Revista de Relaciones Laborales 26: 131-159.

Hernández, J. 2012b. "La inserción laboral de los jóvenes y las características del Empleo juvenil. Diferencias entre la región de Murcia y España". En: El mercado laboral de los jóvenes en la región de Murcia. Murcia, CES.

Hernández, M., y D. López Carmona. 2015. “Jóvenes españoles vulnerables en tiempos de crisis. Un enfoque multidimensional". Aposta. Revista de Ciencias Sociales 67: 9-32.

Hidalgo, A. y S. Pérez. 2004. Aspectos salariales de los jóvenes trabajadores. Madrid: Instituto de la Juventud.

lacovou, M. 2010. "Leaving home: Independence, togetherness and income". Advances in Life Course Research 15(4): 147-160. https://doi.org/10.1016/j.alcr.2010.10.004

Instituto Nacional de Estadística. 2006. Encuesta de Presupuestos Familiares. Consulta 25 de septiembre de 2017 (https://bit.ly/2O4nVye).

Instituto Nacional de Estadística. 2011. Encuesta de Presupuestos Familiares. Consulta 25 de septiembre de 2017 (https://bit.ly/2O4nVye).

Instituto Nacional de Estadística. 2015. Encuesta de Presupuestos Familiares. Consulta 25 de septiembre de 2017 (https://bit.ly/2O4nVye).

Instituto Nacional de Estadística. 2017. Encuesta de Población Activa. Consulta 18 de agosto de 2017 (https://bit. ly/2RwoNOc).

Instituto Nacional de Estadística (2018). Encuesta de Presupuestos Familiares. Consulta 28 de septiembre de 2017 (https://bit.ly/2O4nVye).

Jones, G. (1995). Leaving home. Buckingham: Open University Press.

Jurado, T. (2006). "El creciente dinamismo familiar frente a la ineflexibilidad del modelo de vivienda español". Cuadernos de Información Económica 193: 117-126.

Jurado, T. 2007. "La precariedad temporal-salarial y sus efectos sobre la formación familiar". Sociedad y Utopía 29: 367-403.

Lary, B. 2015. Perceptions of empty nest mothers from diverse socioeconomic backgrounds with boomerang kids. PhD, College of Social and Behavioral Sciences. Walden University. 
Lee, K. O. y G. Painter. 2013. "What happens to household formation in a recession?". Journal of Urban Economics 76: 93-109. https://doi.org/10.1016/j.jue.2013.03.004

López, A. 2005. "Excluidos pero trabajadores: el círculo vicioso de los 'trabajadores con bajo salario' en España”. Cuadernos de Relaciones Laborales 23(1): 153-174.

López, A. 2006. "La familia como respuesta a las demandas de individualización: ambivalencias y contradicciones". Papers. Revista de Sociología 79: 263-284. https://doi. org/10.5565/rev/papers/v79n0.836

Manacorda, M. y E. Moretti. 2006. "Why do most Italian youths live with their parents? Intergenerational transfers and household structure". Journal of the European Economic Association 4(4): 800-829. https://doi. org/10.1162/JEEA.2006.4.4.800

Martínez-Granado, M. y J. Ruiz-Castillo. 2002. "The decisions of Spanish youth: A cross-section study". Journal of Population Economics 15(2): 305-330. https://doi. org/10.1007/s001480100082

Martins, N. y E. Villanueva. 2009. "Does high cost of mortgage debt explain why young adults live with their parents?". Journal of the European Economic Association 7(5): 974-1010. https://doi.org/10.1162/ JEEA.2009.7.5.974

Melo, J. y P. Miret. 2010. "Transición a la vida adulta en España: una comparación en el tiempo y en el territorio utilizando el análisis de entropía". Revista Internacional de Sociología 131: 75-107.

Ministerio de Empleo. 2017. Evolución Salario Mínimo Interprofesional. Consulta 10 de octubre de 2017 (http:// www.empleo.gob.es/estadisticas/bel/SMl/index.htm).

Miret, P. 2006. “Escolarización, mercado de trabajo y emancipación familiar en España: un análisis longitudinal a escala de Comunidad Autónoma". Papeles de Geografía 43: 73-92.

Miret, P. 2007. "Vivir aún en casa o tener casa propia: emancipación residencial en España, 1976-2007". ACE, Arquitectura, Ciudad y Entorno 2(5): 301-330.

Mitchell, B. 2004. "Home, but not alone: Socio-cultural and economic aspects of canadian young adults sharing parental households". Atlantis: Critical Studies in Gender, Culture \& Social Justice 28(2): 115-125.

Moreno, A. 2012a. "The transition to adulthood in Spain in a comparative perspective the incidence of structural factors". Young 20(1): 19-48.
Moreno, A. 2012b. "Transiciones familiares y residenciales". Pp. 52-73 en La transición de los jóvenes a la vida adulta. Crisis económica y emancipación tardía. Barcelona: Fundación La Caixa.

Mykyta, L. 2012. "Economic downturns and the failure to launch: The living arrangements of young adults in the US 1995-2011". Working Paper, 24. US Census Bureau Social, Economic and Housing Statistics Division (SEHSD).

Observatorio de Emancipación. 2009. OBJOVI 26, Primer trimestre de 2009. Consejo de la Juventud de España. Consulta 5 de junio de 2017 (http://www.cje.org/es/publicaciones/publicaciones-periodicas/objovi-26/).

Ortega, A. y P. Martín. 2012. "La juventud española en tiempos de crisis: Paro, vidas precarias y acción colectiva". Sociología del trabajo 75: 93-110.

Pérez-Baleón, G. F. 2012. "Desigualdades de género en el inicio de la vida laboral estable". Papeles de población 18(72): 213-246.

Requena, M. 2002. "Juventud y dependencia familiar en España”. Revista De Estudios De Juventud 58(02): 12-24.

Shanahan, M. J. (2000). "Pathways to adulthood in changing societies: Variability and mechanisms in life course perspective". Annual Review of Sociology 26: 667-692. https://doi.org/10.1146/annurev.soc.26.1.667

Snyder, K. A. 2014. "Globalization and the changing course of adulthood". Contemporary Sociology:A Journal ofReviews 43(2): 183-186. https://doi.org/10.1177/0094306114522414c

South, S. J. y L. Lei. 2015. "Failures-to-launch and boomerang kids: Contemporary determinants of leaving and returning to the parental home". Social Forces 94(2): 863-890. https://doi.org/10.1093/sf/sov064

Vieira, J. M. y Gamundi, P. M. (2010). "Transición a la vida adulta en España: una comparación en el tiempo y en el territorio utilizando el análisis de entropía". Revista Española de Investigaciones Sociológicas (REIS), 131(33): 75-107.

Vosko, L. F. 2010. Managing the margins: Gender, citizenship and the international regulation of precarious employment. New York, NY: Oxford University Press.

White, L. 1994. "Coresidence and leaving home: Young adults and their parents". Annual Review of Sociology 20: 81-102. https://doi.org/10.1146/annurev.so.20.080194.000501

Williams, R. 2012. "Using the margins command to estimate and interpret adjusted predictions and marginal effects". Stata Journal 12(2): 308-331. 
MARTA DONAT LÓPEZ Licenciada en Sociología y máster en Problemas Sociales: Dirección y Gestión de Programas Sociales por la Universidad de Granada. Actualmente, investigadora pre-doctoral y técnica de investigación en el Departamento de Sociología de la Universidad de Granada con un proyecto financiado por el Centro de Estudios Andaluces, mediante el que se está elaborando el diseño del III Plan de Juventud Integral de Andalucía para los próximos 4 años. Ha realizado una estancia de investigación durante 3 meses en 2016-2017 en el German Youth Institute (Múnich, Alemania). Sus líneas de investigación son la juventud, la emancipación y el retorno, las relaciones familiares y la convivencia en el hogar.

Ma DOLORES MARTÍN-LAGOS LÓPEZ Profesora contratada doctora titular de la Universidad de Granada. Ha trabajado en un proyecto de la Delegación del Gobierno para el Plan Nacional de Drogas y ha sido beneficiaria durante cuatro años del contrato de Formación del Profesorado Universitario (FPU). Ha sido técnica de investigación en el Centro de Estudios Andaluces y profesora en la Universidad de Málaga. Ha llevado a cabo estancias predoctorales en el European Monitoring Center for Drugs and Drug Addiction y en el Center for Comparative Research de la Universidad de Stirling, en el programa Marie Curie. Ha sido técnica de investigación visitante en el Oxford Institute of Population Aging. Sus líneas actuales de investigación son la educación, el consumo, los jóvenes y la emancipación.

ELISABETH MOLES LÓPEZ Licenciada en Sociología y máster en Problemas Sociales: Dirección y Gestión en Programas Sociales por la Universidad de Granada. Actualmente está trabajando como técnica de investigación en el Departamento de Sociología de la Universidad de Granada. Ha sido contratada por el Sistema de Garantía Juvenil, financiada por el Fondo Social Europeo. Sus líneas de investigación son la educación, los jóvenes y la emancipación. 